\title{
w-MPS Risk Aversion and the CAPM*
}

\author{
Phelim P. Boyle ${ }^{1}$, Chenghu $\mathrm{Ma}^{2 \#}$ \\ ${ }^{1}$ School of Business and Economics, Wilfrid Laurier University, Waterloo, Canada \\ ${ }^{2}$ School of Management \& Fudan Finance Center, Fudan University, Shanghai, China \\ Email: "machenghu@fudan.edu.cn
}

Received September 29, 2013; revised October 29, 2013; accepted November 6, 2013

Copyright (C) 2013 Phelim P. Boyle, Chenghu Ma. This is an open access article distributed under the Creative Commons Attribution License, which permits unrestricted use, distribution, and reproduction in any medium, provided the original work is properly cited. In accordance of the Creative Commons Attribution License all Copyrights (C) 2013 are reserved for SCIRP and the owner of the intellectual property Phelim P. Boyle, Chenghu Ma. All Copyright (C) 2013 are guarded by law and by SCIRP as a guardian.

\begin{abstract}
This paper establishes general conditions for the validity of mutual fund separation and the equilibrium CAPM. We use partial preference orders that display weak form mean preserving spread (w-MPS) risk aversion in the sense of Ma (2011). We derive this result without imposing any distributional assumptions on asset returns. The results hold even when the market contains an infinite number of securities and a continuum number of traders, and when each investor is permitted to hold some (arbitrary) finite portfolios. A proof of existence of equilibrium CAPM is provided for finite economies by assuming that when preferences are constrained on the market subspace spanned by the risk free bond, the market portfolios admit continuous utility representations.
\end{abstract}

Keywords: CAPM; w-MPS; Risk Aversion; Infinite \& Incomplete Market; Non-Gaussian Returns

\section{Introduction}

This paper provides general conditions for the classical CAPM as an equilibrium model in economies with a frictionless market. First, we show that, if equilibrium exists, then the asset returns must satisfy the CAPM if all investors are risk averse in a sense of mean-preserving spread $^{1}$ (Theorem 1). Second, we prove the existence of equilibrium satisfying the CAPM for economies with w-MPS risk averse investors, without assuming complete markets (Theorem 2). Our results advance the literature and lead to deeper understanding of the extent to which the CAPM holds in equilibrium: 1) preferences satis-

\footnotetext{
*This paper has benefitted from discussions with Jonathan Berk, Bernard Cornet, Darrell Duffie, Editya Goenka, Chiaki Hara, Atsushi Kajii Juan Pedro Gómez, Patrick Leoni, Ning Sun, Dong Chul Won, and Zaifu Yang.

Ma acknowledges financial supports from the Economic and Social Science Research Council, UK (R000223337), the Nature Science Foundation of China $(70871100,71271058)$, and the Fudan Finance Center (EZH4301102/020).

${ }^{\#}$ Corresponding author.

${ }^{1}$ An investor exhibits w-MPS risk aversion if, for all random payoffs $X$ and $Y=X+\varepsilon$, the investor would prefer $X$ to $Y$ whenever $\mathbb{E}[\varepsilon]=0$ and $\operatorname{Cov}(X, \varepsilon)=0$. The notion of mean-preserving-spead used in this paper is weaker than the notion of MPS in $[1,2]$, and is thus denoted w-MPS. Our w-MPS risk averse preferences correspond to the strict variance averse preferences used by [3].
}

fy w-MPS risk aversion, and such preferences can be incomplete and non-transitive; 2) the population space, or investors' type space, can be finite or continuum infinite; and 3) the market may contain a finite, or a countable infinite number of securities, while the market portfolio is composed of a (fixed) finite number of risky assets $^{2}$.

It has been known for a long time that if all investors have mean-variance preferences, then the CAPM holds ([5-7]). It is also known that mean-variance preferences persist for general probabilistic sophisticated expected and non-expected utility functions when asset returns are elliptically distributed ([8,9]). It is, therefore, of particular interest to explore if the CAPM holds when preferences do not fall into the mean-variance class, particularly when asset returns may follow arbitrary distributions $^{3}$. Since the existence of equilibrium CAPM docu-

\footnotetext{
${ }^{2}$ The validity of CAPM, along with existence of equilibrium CAPM, for the case of a finite number of securities and a finite number of investors was documented in [4], Chapter 5. This paper can be thus regarded as a generalization of [4] to infinite economies.

${ }^{3}$ In Duffie's book [3] it contains a derivation of CAPM. Duffie's derivation rests on several explicit and implicit technical assumptions on asset returns: first, the existence of a continuous linear pricing rule in the market span; second, the market subspace is assumed to be a closed subset in $\mathbb{L}_{2}$. Whilst the linearity of the pricing rule is necessarily implied by the no-arbitrage condition as part of the equilibrium conditions, the closedness assumption on the market space is somewhat arbitrary.
} 
mented in literature can be largely built up on the finite specification with respect to the number of tradable securities and the number of investors, it is thus also desirable to consider infinite economies.

In comparison with the above cited work, this paper makes no distributional assumption on asset returns, and purely focuses on investor's risk preferences over the random payoffs. The assumption of $\mathrm{w}$-MPS risk aversion is appealing because 1) it captures investor's psychological aversion towards "increase in risk" in a natural way, 2) w-MPS risk averse preferences are not restricted to certain classes of expected or non-expected utility functions. Therefore, they are not subject to criticisms such as the well-known Allais Paradox and other deficiencies associated with expected utility functions. It is noted that expected utility functions may violate w-MPS risk aversion-and it could even be argued that this constitutes another drawback of expected utility functions. In fact, we shall show that CAPM holds even when investors' preferences are not complete, nor transitive.

The relationship between mean-variance utility functions and the w-MPS risk averse preferences has been analyzed. It is shown in [10] that, when the market contains a finite number of risky assets (but no less than three) and when the market span forms a convex cone of $\mathbb{L}_{2}$ if the w-MPS risk averse preference is represented by continuously Frechét-differentiable (in the $\mathbb{L}_{2}$-norm) utility functions, then the preference must admit a meanvariance utility representation. Nevertheless, one may still want to treat the mean-variance preferences as a subset of the w-MPS risk averse preferences. In fact, a binary relationship satisfying the w-MPS risk aversion property constitutes a partial order, which may not admit an utility representation. As an illustrative example, consider the following Lexicographic binary relation $\succ$ defined on $\mathbb{L}_{\infty}$ : For a given positive integer $n>2$, $X \succ Y$ if $X$ dominates $Y$ by the first order stochastic dominance (i.e., $X \stackrel{\text { dist }}{=} Y+\varepsilon$, where $\varepsilon \geq 0$ and $\varepsilon \neq 0$ ); or, if there exists $2 \leq m \leq n$ such that (a)

$\mathbb{E}\left[X^{i}\right]=\mathbb{E}\left[Y^{i}\right]$ for $0 \leq i<m$, and (b) $(-1)^{m+1} \mathbb{E}\left[X^{m}\right]>(-1)^{m+1} \mathbb{E}\left[Y^{m}\right]$. In this binary relation, in order to rank random payoffs $X$ and $Y$, which may not dominate each other by first order stochastic dominance, one may need to compare their moments up to the $n$th order. The binary relation displays w-MPS risk aversion property, yet it does not admit a mean-variance representation! Indeed, most of the analyses to be carried out in this paper do not rely on the assumption on the existence of a mean-variance utility representation.

Equilibrium with continuum of traders was thoroughly studied in literature ([11-13]). [13] also contains an abstract treatment of equilibrium with incomplete orders. Existence of equilibrium with incomplete and non-transi- tive preferences (with finite number of traders) traces to $[14,15]$. The w-MPS risk averse preferences assumed in this paper is neither complete, nor transitive, thus falls into the category studied in [14]. Also, in this paper we adopt general population type space with arbitrary population mass distribution functions which may accommodate the case of finite, countable infinite, as well as a continuum range of investors.

It is noted that the existing proofs of equilibrium provided in these earlier studies cited above are all with respect to the standard Warasian equilibrium in a context of certainty. In contrast, the existing proof provided in this paper is for a particular stochastic finance economy. Specifically, we assume that the financial market can be incomplete, and that the economy consists of an arbitrary (finite, countable infinite or continuum) number of investors and each investor has (incomplete and non-transitive) a preference order displaying w-MPS risk aversion. The key assumption underlying the existing proof in our paper is that when investor's preference is restricted to the market subspace of efficient portfolios, it admits a continuous utility representation, even though an utility representation over the entire market span may not exist.

From technical point of view, our existing proofs are very much in line with those in the literature $([16,17])$. [16] assumes a finite number of mean-variance investors and a complete market, while [17] assumes a finite number of investors and that each investor has an well-defined utility function defined on the market subspace spanned by the risk free bond and a common risky asset - the market portfolio. The literature contains several other existing proofs for the equilibrium CAPM, all relying crucially on the assumption of mean-variance preferences (See, for instance, [18,19]).

The derivation of CAPM provided in this paper follows the heritage of the traditional approach $([5,6])$. It is based on the relevance of mean-variance efficient frontier to investor's optimal portfolio holdings. Following the standard treatment in literature $([18,20])$ we restrict investors to hold portfolios involving in only a finite number of securities even though the market may contain an infinite number of tradable securities. The finiteness assumption on the market portfolio is not restrictive since, in practice, the market portfolio is an index of a finite number of stocks. Derivative securities written on the stocks and indeces of stocks, which represent a large or even an infinite number of traded financial securities, are not in the composite of the market portfolio. Our model thus provides a useful mechanism to price, which provides not only those primitive securities in the composite of the market portfolio, but also securities out of the composite of the market portfolio.

The remainder of the paper is organized as follows: Section 2 describes the model and summarizes the main 
results. Section 3 introduces the notion of generalized efficient frontiers. Section 3 also discusses w-MPS risk averse investor's optimal portfolio choice problem and its relevance to the generalized efficient frontier. Section 4 includes a formal derivation of the CAPM, along with the two-fund separation theorem. The proofs of the existence of equilibrium are outlined in Section 5. Section 6 concludes the paper.

\section{Outline of the Model and Main Results}

This section describes the basic framework and summarizes the main results of the paper. We start with several useful notations. Let $f$ and $g$ be two real-valued functions (vectors). We write $f \geq g$ if $f(t) \geq g(t)$ for all $t ; f>g$ if $f(t) \geq g(t)$ for all $t$ and $f \neq g$; and $f \gg g$ if $f(t)>g(t)$ for all $t$.

We consider a two-period exchange economy with a frictionless capital market and heterogenous agents. The uncertainty is summarized by a probability state space $(\Omega, \mathbb{P})$ with probability measure $\mathbb{P}$. The topological properties of the state space are otherwise not specified. There exists a countable set of non-redundant risky securities $J=\{1, \cdots, j, \cdots\}$ available for trade ${ }^{4}$. Let $\# J \leq \infty$ be the number of tradable (risky) securities. Security $j$ is associated with a state-contingent random payoff $\delta^{j} \in \mathbb{L}_{2}(\Omega, \mathbb{P})$. Security 0 is a risk free bond with unit payoff in all future states (i.e., $\delta^{0} \equiv 1$ ). Let $p_{j}$ be the price for security $j$. The (total) return on security $j$ is

thus given by $R^{j}=\frac{\delta^{j}}{p_{j}} \in \mathbb{L}_{2}(\Omega, \mathbb{P})$ (if $p_{j} \neq 0$ ). The return for the risk free bond is denoted $R_{f}$.

Let $\Phi \subseteq \mathbb{R}^{\# J}$ be a set of admissible portfolios on $J^{5}$. We restrict $\Phi$ to consist of portfolios that involve only a finite number of risky assets; that is,

$$
\Phi=\left\{\phi \in \mathbb{R}^{\# J}: \sup \left\{j \in J: \phi_{j} \neq 0\right\}<\infty\right\} .
$$

Here, $\phi_{j}$ represents the number of shares in risky security $j$. The market span $\mathbb{D} \subseteq \mathbb{L}_{2}(\Omega, \mathbb{P})$ consists of all possible portfolio payoffs obtainable by trading:

$$
\mathbb{D}=\left\{d \in \mathbb{L}_{2}(\Omega, \mathbb{P}): \exists \phi \in \Phi \text { s.t. } \phi \cdot \delta=d\right\} .
$$

Following the standard literature, we may refer the composite of a portfolio by its weight allocated to each of the risky assets, as well as the number of shares invested in the assets. Precisely, let $\Theta$ be a copy of $\Phi$. Let $W_{0}$ be the initial wealth. Let $\theta \in \Theta$ be a portfolio with $\theta_{j}$ representing the proportion of initial wealth invested in risky security $j$. The number of shares in

\footnotetext{
${ }^{4}$ By non-redundancy, we mean that for each security $j$, its payoff $\delta^{j}$ cannot be duplicated by holding a finite portfolio $\phi \in \Phi$ of other tradable securities.

${ }^{5}$ Much of the analysis below applies for a general admissible set such as $\Theta=\mathbb{H}_{2}(\Sigma)$ defined in Section 3, which may contain portfolios with an infinite number of securities.
}

security $j$ held is thus given by $\frac{\theta_{j} W_{0}}{p_{j}}$, and the amount invested in the risk free bond is $W_{0}(1-\theta \cdot \overline{1})$, where $\overline{1} \in \mathbb{R}^{\# J}$ is the vector of unit elements. For each $\theta \in \Theta$, the portfolio return is $R^{\theta}=R_{f}+\sum_{j \in J} \theta_{j}\left(R^{j}-R_{f}\right)$, with expected portfolio return and standard deviation respectively denoted by $\mu[\theta]$ and $\sigma[\theta]$.

There is a bunch of investors, indexed by $t \in T$. T can be taken as a closed interval, say $[0,1]$, on the Euclidean space $\mathbb{R}$. The population distribution is summarized by a positive measure $\Pi(\cdot)$ on $T$ such that, for each Borel set $B \subseteq T, \Pi(B)$ is the population mass belonging to $B$. Type $t$ investor is endowed with an vector of shares $e(t) \in \Phi$. Investors express preferences over $\mathbb{D}$. The type $t$ investor's preference is summarized by a partial binary relation $\succeq^{t}$ on $\mathbb{D}$.

With these, the exchange economy is summarized by

$$
\mathcal{E}=\left((\Omega, \mathbb{P}), \mathbb{D} ;(T, \Pi),\left\{\succeq^{t}, e(t)\right\}_{t \in T}\right) .
$$

Economy $\mathcal{E}$ is finite if the state space $\Omega$ contains only finite elements; otherwise, it is infinite. For finite economies, it is sensible to assume the number of nonredundant securities is finite and no more than the number of states. Throughout this paper, we make the following assumptions for $\mathcal{E}$ :

A1 $\delta>0$ and the payoffs for the risky securities have a positive definite (infinite-dimensional) covariance matrix $\Sigma_{0}$; that is, for all $x \in \Phi, x^{\top} \Sigma_{0} x \geq 0$, and $x^{\top} \Sigma_{0} x=0 \Leftrightarrow x=\varnothing$.

A2 $\Pi$ satisfies Feller's property: Let $O$ be an open set in $\mathbb{R}$, and $\bar{O}$ be the closure of $O$. For any real-valued integrable function $f$ on $O \times T, y \rightarrow f(y, t)$ to be continuous on $O, \Pi$-a.s., implies $y \rightarrow \int_{T} f(y, t) \Pi(\mathrm{d} t)$ to be continuous on $O$; particularly, for all $y_{0} \in \bar{O}$, $\lim _{y \rightarrow y_{0}} \int_{T} f(y, t) \Pi(\mathrm{d} t)=\int_{T} \lim _{y \rightarrow y_{0}} f(y, t) \Pi(\mathrm{d} t)$ whenever the limit on the right hand side converges ${ }^{6}$.

A3 $e: T \rightarrow \Phi$ is $\Pi$-measurable with $e(t) \cdot \delta>0$. Moreover, $0<\phi^{m}=\int_{T} e(t) \Pi(\mathrm{d} t) \in \Phi$ and $\delta^{m} \stackrel{\Delta}{=} \phi^{m} \cdot \delta>0$.

A4 $\succeq: T \rightarrow \mathbb{D} \times \mathbb{D}$ is $\Pi$-measurable. And, for each $t$, $\succeq^{t}$ is strictly monotonic and homothetic, and displays w-MPS risk aversion in the sense that $X \succeq^{t} Y$ whenever $Y \stackrel{\text { dist }}{=} X+\varepsilon$, where $\mathbb{E}[\varepsilon]=0$ and $\operatorname{Cov}(X, \varepsilon)=0$, and that the preference strictly holds if $\varepsilon \neq 0$.

When trading takes place at time 0 , after observing security prices $p=\left[p_{0}, \cdots, p_{j}, \cdots\right] \in \mathbb{R}^{\# J}$ investor $t$

${ }^{6}$ The Feller's condition is purely technical. It obviously holds true when the economy consists of a finite number of investors. In this case, $\Pi$ corresponds to a measure of finite supports. 
may assess his initial wealth $W_{0}(t)=p \cdot e(t)$ and revise his portfolio holding accordingly. $\phi \in \Phi$ is feasible (for $t)$ if $p \cdot \phi \leq W_{0}(t)$. A feasible allocation $\phi^{*} \in \Phi$ is optimal (for $t$ ) if $\phi^{*} \cdot \delta \succeq_{t} \phi \cdot \delta$ for all $\phi \in \Phi$ that is feasible for $t$. By strict monotonicity of the preference relation, at the optimum, the budget constraint must hold with equality; that is, $p \cdot \phi^{*}=W_{0}(t)$.

If security prices are all non-zero as must be the case when $\delta>0$, the optimality condition can be expressed in terms of portfolio weights $\theta \in \Theta$ allocated to each risky securities. For instance, for $W_{0}(t)>0$, we have $\theta^{*} \in \Theta$ is optimal if, for all $\theta \in \Theta, \quad R^{\theta^{*}} \succeq_{t} R^{\theta}$.

The market equilibrium for $\mathcal{E}$ consists of a price vector $p$ and an asset allocation $\phi^{*}: T \rightarrow \Phi$ that is $\Pi$-measurable such that (i) $\int_{T} \phi^{*}(t) \Pi(\mathrm{d} t)=\phi^{m}$; and (ii) for all $t, \phi^{*}(t)$ is optimal w.r.t. $p$ for type $t$ investors.

Again, for the case when equilibrium security prices are all non-zero, and when investors' initial wealth at $p$ are all positive, the equilibrium condition can be equivalently stated in terms of portfolio allocation $\theta^{*}: T \rightarrow \Phi$ with condition (i) replaced by $\int_{T} \frac{\theta_{j}^{*}(t) W_{0}(t)}{p_{j}} \Pi(\mathrm{d} t)=\phi_{j}^{m}$ for all $j$, in addition to the optimality condition (in term of $\theta^{*}$ ) for all type investors.

Let $\theta^{m} \in \Theta$ be the market portfolio with $\theta_{j}^{m}=\frac{\phi_{j}^{m} p_{j}}{\phi^{m} \cdot p}, j \in J$. Let $R_{m}$ be the return of the market portfolio, and let $\mu_{m}=\mathbb{E}\left[R_{m}\right]$. We state the first main result of the paper:

Theorem 1 Suppose conditions A1-A4 hold. If the economy achieves its equilibrium at $p$ (with $p_{j} \neq 0$ for all $j$ ) with equilibrium portfolio allocation $\theta^{*}$, then

(1) there exists $\alpha: T \rightarrow \mathbb{R}$, that is $\Pi$-measurable and satisfies $\int_{T} \alpha(t) \Pi(\mathrm{d} t)=1$, such that $\theta^{*}(t)=\alpha(t) \theta^{m}$ for all $t \in T$;

(2) the capital asset pricing model (CAPM) holds

$$
\mu[\theta]=R_{f}+\beta^{\theta}\left(\mu_{m}-R_{f}\right), \forall \theta \in \Theta
$$

where $\beta^{\theta}=\frac{\operatorname{Cov}\left(R^{\theta}, R_{m}\right)}{\operatorname{Var}\left(R_{m}\right)}$.

Theorem 1-(1) is about mutual fund separation of equilibrium allocation-investors of all type optimally hold a proportion of the market portfolio, in addition to a position in the risk free bond. It extends the well known Black's two-fund separation theorem [21] to this infinite economy. The proof of the statement is based on insight on the mathematical properties of the efficient frontier covered in Section 3. The notion of efficient frontier, which is formally introduced below in Definition 3.1, is a direct extension to that of Markowitz ([22,23]) by considering the possibility of an infinite number of tradable securities. Proof of the equilibrium CAPM outlined in Theorem 1-(2) is summarized in Section 4.

To find an equilibrium price vector, we work with a set of "normalized prices" satisfying the CAPM, and the term "normalized prices" will be made precise in Section 5. Let $\Delta \subseteq \mathbb{R}^{\# J}$ be the set of normalized price vector satisfying the CAPM. We shall show that the set $\Delta$ forms a straight line on the vector space $\mathbb{R}^{\# J}$ (Re: Lemmas 4 and 5), and that, for each $p \in \Delta$, investor's optimal portfolio, if it exists, must be expressed as a combination of the risk free bond and the market portfolio (Re: Lemma 8).

Denote by $\mathbb{D}_{0, m} \subseteq \mathbb{D}$ the market subspace spanned by the risk free bond and the market portfolio. We write

$$
\mathbb{D}_{0, m}=\left\{x+y \delta^{m}: x, y \in \mathbb{R}\right\} .
$$

Recall that, by assumption $\mathrm{A} 3, \phi^{m}$ is finite and $\delta^{m} \in \mathbb{L}_{\infty}(\Omega, \mathbb{P})$ is bounded. To prove the existence of equilibrium, we assume further that investor's preference restricted on $\mathbb{D}_{0, m}$ admits an utility representation ${ }^{7}$. Put this formally

A5 For each $t$, the restriction of $\succeq^{t}$ on $\mathbb{D}_{0, m}$ admits a utility representation: $U_{t}: \mathbb{D}_{0, m} \rightarrow \mathbb{R}$. And, the utility function $(x, y) \rightarrow U_{t}\left(x+y \delta^{m}\right)$ is continuous and strictly quasi-concave.

Assumption A5 is logically consistent with the w-MPS risk averse behavioral assumption (A4) since there exists no w-MPS dominating relations for each bundles in $\mathbb{D}_{0, m}$. The second main result of this paper concerns the existence of equilibrium CAPM.

Theorem 2 Suppose $\mathcal{E}$ is finite and satisfies A1-A5. Then, the equilibrium exists and, at equilibrium, the CAPM holds.

The proof of Theorem 2 is contained in Section 5. For the validity of Theorem 2, we may replace A5 with A5' below:

A5' The restriction of $\succeq^{t}$ on the market subspace spanned by those efficient portfolios (if exists) admits a utility representation.

In fact, by Lemma 8, we see that A5' implies A5 when security prices are governed by the CAPM.

\section{MV Analysis and Portfolio Choice}

This section starts with a discussion of mean-variance efficiency and generalized mean-variance efficiency as an infinite dimensional extension to Markowitz meanvariance efficiency. It follows with an in-depth discussion on the relevance of the mean-variance efficiency to

\footnotetext{
${ }^{7}$ Since payoffs within the market subspace do not display mean preserving spead to each other, assumption A5 is thus logically consistent with assumption A4.
} 
optimal portfolio choice by w-MPS risk averse investors.

\subsection{Efficient Frontier}

Taking as given an arbitrary price vector $p \in \mathbb{R}^{\# J}$ with $p_{j} \neq 0$ for all $j$ so that the asset returns are well-defined for all tradable securities. Let $\mathbb{X} \subseteq \mathbb{R}^{\# J}$ be an admissible portfolio space. For each $\theta \in \mathbb{X}$, the portfolio return has its mean return and standard deviation respectively given by

$$
\mu[\theta]=R_{f}+\theta^{\top}\left(\mu-R_{f} \overline{1}\right), \sigma[\theta]=\left(\theta^{\top} \Sigma \theta\right)^{\frac{1}{2}},
$$

where $\mu$ and $\Sigma$ represent the vector of expected returns and the variance-covariance matrix of the risky returns. Similarly, for two arbitrary risky portfolios $\theta$ and $\theta^{\prime}$, the covariance of the two portfolio returns is $\sigma\left[\theta, \theta^{\prime}\right]=\theta^{\top} \Sigma \theta^{\prime}$. For pure risky portfolios $\theta$ we have $\theta \cdot 1=1$.

The following definition of efficient portfolio and efficient frontier for $\mathbb{X}$ applies whether or not there is a risk free asset.

Definition 1 For $\mu_{0} \in \mathbb{R}, \theta_{0} \in \mathbb{X}$ is said to be efficient at $\mu_{0}$ if $\theta_{0}=\arg \min _{\theta \in \mathbb{X}}\left\{\sigma[\theta]: \mu[\theta]=\mu_{0}\right\}$. The curve

$$
\mathcal{I}(\mathbb{X})=\{(\mu[\theta], \sigma[\theta]): \theta \in \mathbb{X} \text { is efficient }\}
$$

is referred to as the mean-variance efficient frontier, or simply the "efficient frontier", with respect to $\mathbb{X}$.

The next proposition describes a general property of efficient portfolios.

Proposition 1 Let $\theta_{0} \in \mathbb{X}$ be an efficient portfolio with mean $\mu_{0}$. For all $\theta \in \mathbb{X}$ with $\mu[\theta]=\mu_{0}$, we have: $R^{\theta}=R^{\theta_{0}}+\varepsilon$ with $\mathbb{E}[\varepsilon]=0$ and $\operatorname{Cov}\left(R^{\theta_{0}}, \varepsilon\right)=0$.

Proof. Consider the set of portfolios $\left\{\alpha \theta+(1-\alpha) \theta_{0}: \alpha \in \mathbb{R}\right\}$ formed by convex combinations of $\theta_{0}$ and $\theta$. These portfolios all have the same mean return given by $\mu_{0}$ Since $\theta_{0}$ is efficient at $\mu_{0}$ with standard deviation $\sigma_{0}, \sigma\left[\alpha \theta+(1-\alpha) \theta_{0}\right]$ must achieve its minimum at $\alpha=0$; that is,

$$
0=\arg \min _{\alpha}\left\{\alpha^{2} \sigma^{2}[\theta]+2 \alpha(1-\alpha) \sigma\left[\theta, \theta_{0}\right]+(1-\alpha)^{2} \sigma_{0}^{2}\right\}
$$

The first order condition leads to $\sigma\left[\theta, \theta_{0}\right]=\sigma_{0}^{2}$. Let $\varepsilon=R^{\theta}-R^{\theta_{0}}$. We have: $\mathbb{E}[\varepsilon]=0$ and $\operatorname{Cov}\left(R^{\theta_{0}}, \varepsilon\right)=0$.

Notice that this proof does not require assumption on the finiteness of the number of securities, nor requires the covariance matrix $\Sigma$ to be non-singular.

Since investors are restricted to hold finite portfolios, we shall naturally pay special attention to the efficient frontier $\mathcal{I}(\Theta)$ with respect to $\mathbb{X}=\Theta$. In contrast to the finite dimensional case originally considered by Markowitz in $[22,23]$, we do encounter one technical difficulty; that is, when the number of tradable securities is infinite the efficient frontier $\mathcal{I}(\Theta)$ could be empty and thus not well defined.

There is another case that is relevant to choices made by w-MPS risk averse investors, and is of particular interest. This refers to the efficient frontier for $\mathbb{X}=\mathbb{H}_{2}(\Sigma)$. Here,

$$
\mathbb{H}_{2}(\Sigma)=\left\{\theta \in \mathbb{R}^{\# J}: \theta^{\top} \Sigma \theta<\infty\right\}
$$

is an Hilbert space with inner product $\left\langle\theta, \theta^{\prime}\right\rangle_{\Sigma}=\theta^{\top} \Sigma \theta^{\prime}$ and norm $\|\theta\|_{\Sigma}=\sqrt{\langle\theta, \theta\rangle_{\Sigma}}$. The portfolio space $\Theta$ forms a dense but not closed subset of $\mathbb{H}_{2}(\Sigma)$. Since elements in $\mathbb{H}_{2}(\Sigma)$ are with possibly infinite number of securities, the efficient frontier w.r.t. $\mathbb{H}_{2}(\Sigma)$, denoted $\mathcal{I}_{g}$, is called the generalized efficient frontier (g.e.f.).

Unlike $\mathcal{I}(\Theta)$, we shall show that $\mathcal{I}_{g}$ is well defined under fairly general conditions (see Proposition 3). Similar to the finite dimensional case, an analytic expression for the g.e.f. can be obtained. As it turns out, $\mathcal{I}_{g}$ forms a hyperbola on the $\mu-\sigma$ plane in absence of risk free asset. And, in presence of risk free asset, $\mathcal{I}_{g}$ is well defined when the mean return for the minimum risk portfolio differs from the risk free interest rate. For this latter case, $\mathcal{I}_{g}$ is composed of the generalized tangent ray and the reflection of the generalized tangent ray. Accordingly, the classical Black-Tobin mutual fund separation theorems (see [21] and [24]) extend to this infinite dimensional setting for $\mathbb{H}_{2}(\Sigma)$.

\subsection{Portfolio Choice}

When there is a finite number of a risky asset, it is well known that the optimal portfolio for mean-variance investors, if it exists, must be located on the Markowitz mean-variance efficient frontier. The existing literature tells us little about the relevance of efficient frontier for portfolio choices made by investors whose preferences are not in the mean-variance class ${ }^{8}$. The difficulties in establishing such relevancy are well known: First, an investor's optimal portfolio may not exist even though the efficient frontier is well defined. This occurs, for example, when the security prices violate the no-arbitrage conditions which are necessary for the existence of an optimal portfolio for all investors with increasing and continuous utility functions. Second, even if optimal portfolio exists, the efficient portfolio with mean return corresponding to that of the optimal portfolio may not exist. This occurs, for instance, when the mean return of the minimum variance portfolio is equal to the risk free rate. Finally, when the optimal portfolio and efficient portfolio both exist, it is still not obvious if the investor would choose to optimally hold the efficient portfolio because

\footnotetext{
${ }^{8}$ See $[25,26]$ for results on two-fund separation for investors with risk averse expected utility functions. They prove that, the separating portfolios, if they exist, must be on the efficient frontier. See also [27] for conditions on asset returns and the expected utility functions that are sufficient for both two-fund separation and the CAPM.
} 
the investor may care about higher moments beyond the first two. In this section, we study the optimal choice behavior for risk averse investors and explore the relevance of the efficient portfolios in their optimal choices. Here, we restrict our attention to investors who are risk averse in the sense of w-MPS risk aversion.

Recalling first the definition of w-MPS risk aversion. An investor is said to display w-MPS risk aversion if $X \succeq Y$ whenever $Y \stackrel{\text { dist }}{=} X+\varepsilon$, where $\mathbb{E}[\varepsilon]=0$ and $\operatorname{Cov}(X, \varepsilon)=0$. The strict preference holds if $\varepsilon \neq 0$. In this case, we say that $Y$ is identical in distribution to a w-MPS of $X$.

For w-MPS risk averse investors, our next result follows as a corollary to Proposition 1.

Proposition 2 Let $\theta^{*}$ be an optimal portfolio holding for a $w$-MPS risk averse investor. Let $\mu^{*}$ be the portfolio mean return for the optimal portfolio $\theta^{*}$. Then, if the efficient portfolio at $\mu^{*}$ exists, the optimal portfolio must be efficient.

It is well known that, with a finite number of securities, efficient frontiers are well-defined and all efficient portfolios can be expressed as a convex combination of two efficient portfolios. The optimal portfolio holdings for w-MPS risk averse investors can be easily characterized because they would have to be located on the efficient frontier. When investors face an infinite number of investment opportunities $(\# J=\infty)$, it is not clear if mutual fund separation holds. In fact, as to be illustrated below, in presence of infinite number of risky assets, the efficient frontier for $\Theta$ is generically not defined (nonexistence), and the optimal portfolio correspondence (valued in $\Theta$ ) for the w-MPS risk averse investors is generically empty.

We first consider the case when the market contains purely risky portfolios. We impose the following two conditions on the coefficients ${ }^{9}$ :

C1 $\Sigma$ is positive definite, and $\Sigma^{-1} \mu, \Sigma^{-1} \overline{1} \in \mathbb{H}_{2}(\Sigma)$;

C2 Non-degeneracy: $\mu$ is not proportional to 1 .

Under conditions $\mathrm{C} 1$ and $\mathrm{C} 2$, the g.e.f

$\mathcal{I}_{g}=\mathcal{I}\left(\mathbb{H}_{2}(\Sigma)\right)$ is well defined, and the following important risk decomposition result holds for the generalized portfolios:

Proposition 3 Suppose conditions $C 1$ and $C 2$ hold. For all $\theta \in \mathbb{H}_{2}(\Sigma)$, there exists a unique $\theta_{0} \in \mathcal{I}_{g}$ such that $R^{\theta}$ be expressed as a w-MPS of $R^{\theta_{0}}$. Moreover, for all $\mu_{0}$, the generalized efficient portfolio at $\mu_{0}$ is given by

\footnotetext{
${ }^{9}$ We consider normalized securities that forms an orthonormal basis of the market span $\mathbb{D}$. Each normalized security is a finite portfolio in $J$. The set of normalized securities are uncorrelated to each other, and they generate the same market span $\mathbb{D}$ from the original set of securities $J$. For normalized securities, $\Sigma$ is diagonal with positive diagonal elements, its inverse $\Sigma^{-1}$ is also well defined and diagonal.
}

$\theta_{0}=\left[\Sigma^{-1} \mu, \Sigma^{-1} e\right] A^{-1}\left[\mu_{0}, 1\right]^{\top} \in \mathbb{H}_{2}(\Sigma)$ where $A \stackrel{\Delta}{=}[\mu, e]^{\top} \Sigma^{-1}[\mu, e]$ is a $2 \times 2$ positive definite matrix.

Proof. The first statement follows the same argument as in Proposition 1. Condition $\mathrm{C} 2$ implies $\Sigma^{-1} e$ is not proportional to $\Sigma^{-1} \mu$, which in turn implies $\left\langle\Sigma^{-1} e, \Sigma^{-1} \mu\right\rangle_{\Sigma}<\left\|\Sigma^{-1} e\right\|_{\Sigma} \times\left\|\Sigma^{-1} \mu\right\|_{\Sigma}$, and matrix $A$ has a well-defined inverse $A^{-1}$. The quadratic optimization problem

$$
\min _{\theta \in \mathbb{H}_{2}(\Sigma)}\left\{\|\theta\|_{\Sigma}^{2}:\left\langle\Sigma^{-1} e, \theta\right\rangle_{\Sigma}=1 \text { and }\left\langle\Sigma^{-1} \mu, \theta\right\rangle_{\Sigma}=\mu_{0}\right\}
$$

can be readily solved with the Lagrangean method, and the optimal solution is given by Equation (7).

Notice that, the risk-decomposition theorem holds for all generalized risky portfolios in $\mathbb{H}_{2}(\Sigma)$, thus in particular for risky portfolios in $\Theta \subset \mathbb{H}_{2}(\Sigma)$. That is, return for each risky portfolio $\theta$ in $\Theta$ must admit as a w-MPS to that of a generalized efficient portfolio $\theta_{0} \in \mathbb{H}_{2}(\Sigma)$ on $\mathcal{I}_{g}$. This implies that w-MPS risk averse investors would tend to hold generalized efficient portfolios if they were allowed to hold an infinite number of securities. In other words, investors would, in general, not be satiated with holding any arbitrary finite number of securities ${ }^{10}$. This results in (generic) non-existence of optimal portfolio holdings for w-MPS risk averse investors who are restricted to hold finite portfolios in $\Theta$.

Secondly, we consider the case when the market contains a risk free bond, and maintain conditions $\mathrm{C} 1$ and $\mathrm{C} 2$ for the risky assets. Let $\underline{\mu}=\frac{\left\langle\Sigma^{-1} \overline{1}, \Sigma^{-1} \mu\right\rangle_{\Sigma}}{\left\|\Sigma^{-1} \overline{1}\right\|_{\Sigma}^{2}}$. If $\underline{\mu} \neq R_{f}$, then the generalized tangent portfolio would be welldefined and be given by

$$
\theta_{m}=\frac{\Sigma^{-1} \mu-R_{f} \Sigma^{-1} \overline{1}}{\left\langle\Sigma^{-1} \overline{1}, \Sigma^{-1} \mu\right\rangle_{\Sigma}-R_{f}\left\|\Sigma^{-1} \overline{1}\right\|_{\Sigma}^{2}} .
$$

When $\theta_{m}$ contains an infinite number of non-zero elements (that is, $\theta_{m} \notin \Theta$ ), all generalized efficient portfolios (except the risk free bond) would not be admissible. This causes the generic non-existence of optimal portfolios for w-MPS risk averse investors who are restricted to hold finite portfolios. For the extreme case when the generalized tangent portfolio is finite (that is, $\theta_{m} \in \Theta$ ),

\footnotetext{
${ }^{10}$ However, there are two exceptions to this statement: (a) when $\# J<\infty$, the g.e.f. $\mathcal{I}_{g}$ reduces to the Markowitz efficient frontier, and all generalized efficient portfolios become efficient; (b) when $\# J=\infty$ and the (normalized) risky assets contain just a finite number distinct expected returns.

${ }^{11}$ Here, $\mu$ is the mean return for the generalized minimum variance portfolio $\underline{\theta}=\frac{\Sigma^{-1} \overline{1}}{\left\|\Sigma^{-1} \overline{1}\right\|_{\Sigma}^{2}}$
} 
the tangent ray and its reflection constitute the efficient frontier w.r.t. $\Theta$ in presence of an risk free bond.

Based on this discussion, the optimal portfolio holdings for w-MPS risk averse investors can be characterized.

Proposition 4 Suppose conditions $C 1$ and $C 2$ hold. Consider an investor with monotonic and w-MPS risk averse preference.

a) The investor's optimal portfolio, if it exists, must have an expected return no less than the risk free interest rate, $\mu^{*} \geq R_{f}$.

b) When $\mu \neq R_{f}$, the optimal portfolio, if it exists, must be on the generalized efficient rays and be expressed as a combination of the risk free bond and the generalized tangent portfolio $\theta_{m}$. In this case, the generalized tangent portfolio must be finite; that is, $\theta_{m} \in \Theta$.

c) When $\mu=R_{f}$, the optimal portfolio, if not risk free, does not exist.

If the investor were allowed to hold an infinite number of securities, say $\mathbb{X}=\mathbb{H}_{2}(\Sigma)$, the optimal portfolio would exist and be expressed as a combination of the risk free bond and the generalized tangent portfolio. Since the generalized tangent portfolio may not be finite, hence does not belong to $\Theta$, the optimal demand correspondence in $\Theta$ for an w-MPS risk averse investor can be empty.

\section{Proof of Theorem 1}

This section builds upon our earlier results to derive the CAPM with w-MPS risk averse investors. Recall that investors have homogeneous beliefs and so they will perceive the same generalized efficient frontier as described in the previous section. Further to the two-fund separation property (Proposition 4-(b)), to prove the validity of the CAPM we shall first show that, in equilibrium, (a) the generalized tangent portfolio exists and belongs to $\Theta$, and (b) the generalized tangent portfolio coincides with the market portfolio.

Lemma 1 Existence of equilibrium implies $\mu \neq R_{f}$.

Proof. Suppose to the contrary that the equilibrium exists with $\mu=R_{f}$. By Proposition 4-(c), the optimal portfolio would be either given by the risk free bond, or not exist. Since all investors investing in the risk free bond will necessarily violate the market clearing conditions for the risky assets, we thus conclude that, the optimal portfolios do not exist for at least one investor. The latter contradicts the assumption on the existence of equilibrium. Therefore, in equilibrium, we must have $\mu \neq R_{f}$.

Since $\mu \neq R_{f}$, in equilibrium the generalized tangent portfolio $\bar{\theta}_{m}$ is well defined. We can further identify the generalized tangent portfolio to coincide with the market portfolio (in equilibrium) following the standard separating portfolio arguments. So, we may state without proof the following claim.
Lemma 2 Suppose economy $\mathcal{E}$ has an equilibrium that is supported by non-zero equilibrium security prices ( $p_{j} \neq 0$ for all $j \in J$ ). The equilibrium generalized tangent portfolio must be finite $\left(\theta_{m} \in \Theta\right)$; and, in particular, it must be given by the market portfolio; that is, $\theta_{m}=\theta^{m 12}$.

The following risk decomposition theorem is an infinite-dimensional generalization to that of Huang and Litzenberger [26], Chapter 3.18 \& 3.19.

Lemma 3 Suppose $\mu \neq R_{f}$, the generalized tangent portfolio $\theta_{m}$ is well defined; in particular, for all $\theta \in \mathbb{H}_{2}(\Sigma)$ it holds true that ${ }^{13}$

$$
R^{\theta}=R_{f}+\frac{\left\langle\theta_{m}, \theta\right\rangle_{\Sigma}}{\left\|\theta_{m}\right\|_{\Sigma}^{2}}\left(R^{\theta_{m}}-R_{f}\right)+\varepsilon^{\theta}
$$

where $\varepsilon^{\theta}$ has a zero mean and is uncorrelated with $R^{\theta_{m}}$; in particular,

$$
\mu[\theta]=R_{f}+\frac{\left\langle\theta_{m}, \theta\right\rangle_{\Sigma}}{\left\|\theta_{m}\right\|_{\Sigma}^{2}}\left(\mu\left[\theta_{m}\right]-R_{f}\right) .
$$

Lemmas 1, 2 and 3 together lead to the equilibrium CAPM that is valid for all generalized portfolios, particularly for those admissible finite portfolios in $\Theta$ as a subset of $\mathbb{H}_{2}(\Sigma)$. This concludes the validity to Theorem 1-(2).

Since, in equilibrium, the generalized tangent portfolio is given by the market portfolio which, by assumption $\mathrm{A} 3$, is finite, the equilibrium efficient frontier for $\Theta$ with $\# J=\infty$ (and with a risk less asset) is well-defined and is given by the tangent ray and its reflection ray. This, in turn, implies (by Proposition 4-(b)) the validity of twofund separation; that is, in equilibrium, each investors optimally holds a portfolio that involves a combination of the risk free bond and the market portfolio. This confirms the validity of Theorem 1-(1).

Notice further that the CAPM remains valid as an equilibrium model if w-MPS risk averse investors are allowed to hold an infinite number of securities, say portfolios belonging to $\mathbb{H}_{2}(\Sigma)$. This is because, under conditions $\mathrm{C} 1$ and $\mathrm{C} 2$, the efficient frontier w.r.t. $\mathbb{H}_{2}(\Sigma)$ is well-defined, and because w-MPS risk averse investors would all invest in the risk-free asset and the efficient tangent portfolio. Following the same argument as in Lemma 3, the tangent portfolio must coincide with the

\footnotetext{
${ }^{12}$ We can further show that, if equilibrium exists with $W_{0}^{i}>0$ for all $i$, then we must have $\mu_{m}>\underline{\mu}>R_{f}$ and the market portfolio $\theta^{m}$ must be on the efficient ray.

${ }^{13}$ Notice that, when $p_{j} \neq 0$ for all $j$, the risky returns have a nonsingular variance-covariance matrix as long as the covariance matrix for the risky payoffs $\delta$ is non-singular. This last assumption is to ensure that none of the tradable securities are redundant and that $\left\|\theta_{m}\right\|_{\Sigma}>0$.
} 
market portfolio ${ }^{14}$. This would let us conclude the validity of the CAPM along with the two-fund separation for $\mathbb{H}_{2}(\Sigma)$.

\section{Existence of Equilibrium}

We now proceed with the proof of the existence of equilibrium (Theorem 2). We assume finite economies with a finite number of states and a finite number of assets $(\# J<\infty)$.

To find the equilibrium, we shall restrict security prices to satisfy the CAPM.

Lemma 4 If equilibrium exists, then there exists $\pi_{0}>0$ and $\pi_{1}>0$ such that, for all $x \in \mathbb{D}$

$$
\Psi(x)=\mathbb{E}[\pi x] \text { with } \pi=\pi_{0}-\pi_{1} \hat{c},
$$

where $\hat{c}=\frac{\delta^{m}-\mathbb{E}\left[\delta^{m}\right]}{\sigma\left[\delta^{m}\right]}, \pi_{0}=\Psi(1)>0$ and $\pi_{1}=\frac{\Psi(1) \mathbb{E}\left[\delta^{m}\right]-\Psi\left(\delta^{m}\right)}{\sigma\left[\delta^{m}\right]}>0$.

The CAPM pricing rule in Lemma 4 is well known (see, for instance, [3]). Here, $\pi$ is a discount factor. Therefore, given the random payoff for the market portfolio, and given the expressions for $\pi_{0}$ and $\pi_{1}$, the equilibrium pricing rule is fully determined by $\Psi(1)$ and $\Psi\left[\delta^{m}\right]$, which are the prices of the risk free bond and that of the market portfolio.

Lemma 5 If $\pi$ is an equilibrium discount factor, then for all positive constant $k>0, k \pi$ is also an equilibrium discount factor.

By Lemmas 4 and 5, we can normalize the equilibrium discount factor in (11) to be such that $\pi_{0}+\pi_{1}=1$ and write $\pi=\pi(r)$ for some $r \in[0,1]$, where

$\pi(r)=1-r-r \hat{c}$. Denote by $\Psi(r, x)$ the pricing rule resulting from $\pi(r)$. Let $\Psi(r, \delta)$ be the positive price vector for the $J$ risky assets.

To prove the existence of equilibrium, we need to show that there exists an $r$ such that, given prices determined by (11) with $\pi=\pi(r)$, the optimal portfolio exists for each investor and satisfies the market clearing conditions.

To ensure existence of an optimal portfolio, we shall restrict $r$ so that the pricing rule (11) satisfies the noarbitrage condition. By the fundamental theorem of finance (see [28]), the no-arbitrage condition is equivalent to the existence of $v(r) \in \mathbb{L}_{2}(\Omega, \mathbb{P})$ such that $\pi(r)+v(r) \gg 0$ and $v(r)$ is orthogonal to the market span $\mathbb{D}^{15}$. For the case when $\pi(r) \gg 0$, we can choose

\footnotetext{
${ }^{14}$ Here, of course, we must assume $\theta^{m} \in \mathbb{H}_{2}(\Sigma)$.

${ }^{15}$ We see that $v \in \mathbb{L}_{2}(\Omega, \mathbb{P})$ is orthogonal to $\mathbb{D} \subseteq \mathbb{L}_{2}(\Omega, \mathbb{P})$ if $\mathbb{E}[v d]=0$ for all $d \in \mathbb{D}$. We write $v \in \mathbb{D}^{\perp}$ whenever $v$ is orthogonal to $\mathbb{D}$. The set $\mathbb{D}^{\perp}$ is the orthogonal complement of $\mathbb{D}$.
}

$v(r)=0$.

Let $O$ be the subset of $[0,1]$ for which no-arbitrage condition holds. We have

Lemma 6 The set $O \backslash\{0\}$ is an open convex subset of $[0,1]$; in particular, we can write $O=\left[0, r^{*}\right)$ for some $0<r^{*} \leq 1 \notin O$.

Proof. The proof proceeds in five steps. First, $0 \in O$ with $\pi(0)=1$ and $v=0$. Second, $1 \notin O$. This is because, with $\pi=\pi(1)=-\hat{c}$, the price for the market portfolio is negative: $\Psi\left(1, \delta^{m}\right)=-\sigma\left[\delta^{m}\right]<0$. This violates the no-arbitrage condition since, by assumption A3, $\delta^{m}>0$. Third, there exists $r \in(0,1)$ s.t. $r \in O$. Since $\mathcal{E}$ is a finite, $\delta^{m}$ takes finite possible values, and has a finite $\mathbb{L}_{\infty}$-norm, denoted $\left\|\delta^{m}\right\|_{\infty}$. Since $\delta^{m}$ is risky, we have $\left\|\delta^{m}\right\|_{\infty}>\mathbb{E}\left[\delta^{m}\right]$. Let

$r_{m} \stackrel{\Delta}{=} \frac{\sigma\left[\delta^{m}\right]}{\sigma\left[\delta^{m}\right]+\left\|\delta^{m}\right\|_{\infty}-\mathbb{E}\left[\delta^{m}\right]} \in(0,1)$. For all $r \in\left(0, r_{m}\right)$

we have

$$
\begin{aligned}
\pi(r) & =1-r\left(1+\frac{\delta^{m}-\mathbb{E}\left[\delta^{m}\right]}{\sigma\left[\delta^{m}\right]}\right) \\
& \geq 1-r\left(1+\frac{\left\|\delta^{m}\right\|_{\infty}-\mathbb{E}\left[\delta^{m}\right]}{\sigma\left[\delta^{m}\right]}\right)>0 ;
\end{aligned}
$$

that is, $\pi(r) \gg 0$ with $v(r)=0$. Fourth, we show that $O$ is connected: Let $r \in O$ with $\pi(r)+v(r) \gg 0$ and $v(r) \in \mathbb{D}^{\perp}$. For any $a \in(0,1)$ we have $a v(r) \in \mathbb{D}^{\perp}$ and $\pi(a r)+a v(r)=1-a+a\{\pi(r)+v(r)\} \gg 0$. So,

ar $\in O$. Finally, $O \backslash\{0\}$ is open. For any $0<r_{0} \in O$ with $\pi\left(r_{0}\right)+v\left(r_{0}\right) \gg 0$ and $v\left(r_{0}\right) \in \mathbb{D}^{\perp}$. For $\epsilon>0$ sufficiently small, set $v(r)=v\left(r_{0}\right)$ for all $r \in\left(r_{0}-\epsilon, r_{0}+\epsilon\right)$. We have

$$
\begin{aligned}
& \pi(r)+v(r) \\
& =\pi\left(r_{0}\right)+v\left(r_{0}\right)-\left(r-r_{0}\right) \hat{c} \geq \pi\left(r_{0}\right)+v\left(r_{0}\right)-\epsilon\|\hat{c}\|_{\infty} .
\end{aligned}
$$

Since $\pi\left(r_{0}\right)+v\left(r_{0}\right)$ is strictly positive and takes finite values, when $\epsilon$ is sufficiently small, the right hand side, namely $\pi\left(r_{0}\right)+v\left(r_{0}\right)-\epsilon\|\hat{c}\|_{\infty}$, must be strictly positive. This yields $\pi(r)+v(r) \gg 0$. Consequently, we can write $O=\left[0, r^{*}\right)$ for some $0<r^{*} \in(0,1]$

We understand that, the no-arbitrage condition is violated at $r^{*}$. For all $r \in O$, let $\theta_{m}(r)$ and $\theta^{m}(r)$ be respectively the generalized tangent portfolio and the market portfolio. For all $x \in \mathbb{R}^{\# J}$, let $\operatorname{diag}[x]$ be the diagonal matrix with $j$ th diagonal element given by $x_{j}$.

Lemma 7 For all $0<r \in O$, the generalized tangent portfolio $\theta_{m}(r)$ is well-defined and is given by

$$
\theta_{m}(r)=\frac{\operatorname{diag}[\Psi(r, \delta)] \Sigma_{0}^{-1}\left(\mathbb{E}[\delta]-\Psi^{-1}(r, 1) \Psi(r, \delta)\right)}{\Psi^{\top}(r, \delta) \Sigma_{0}^{-1}\left(\mathbb{E}[\delta]-\Psi^{-1}(r, 1) \Psi(r, \delta)\right)} .
$$


Proof. Since all risky securities are with non-negative payoffs, the no-arbitrage condition implies the price vector $\Psi(r, \delta)$ to be strictly positive. By assumption A1, $\Sigma_{0}$ is positive definite, so the co-variance matrix $\Sigma$ for asset returns is well defined, and is positive definite. We can further verify that, for all $0<r \in O$,

$\mu(r) \neq \Psi^{-1}(r, 1)=R_{f}$. This in turn implies, by Proposition 4 , the existence of the efficient rays with a well defined generalized tangent portfolio given by

$\theta_{m}(r)=\frac{\Sigma^{-1}\left(\mu-R_{f} \overline{1}\right)}{\left\langle\Sigma^{-1} \overline{1}, \Sigma^{-1} \mu\right\rangle_{\Sigma}-R_{f}\left\|\Sigma^{-1} \overline{1}\right\|_{\Sigma}^{2}}$ in which

$\left\langle\Sigma^{-1} \overline{1}, \Sigma^{-1} \mu\right\rangle_{\Sigma}-R_{f}\left\|\Sigma^{-1} \overline{1}\right\|_{\Sigma}^{2} \neq 0$ for $0<r \in O$. The desired expression for the generalized tangent portfolio is valid because

$$
\begin{aligned}
& \Sigma^{-1}=\operatorname{diag}[\Psi(r, \delta)] \Sigma_{0}^{-1} \operatorname{diag}[\Psi(r, \delta)] \\
& \mathbb{E}[\delta]-\frac{\Psi(r, \delta)}{\Psi(r, 1)}=\operatorname{diag}[\Psi(r, \delta)]\left(\mu-R_{f} \overline{1}\right) \\
& \Psi^{\top}(r, \delta)=\overline{1}^{\top} \operatorname{diag}[\Psi(r, \delta)] .
\end{aligned}
$$

We can further show that

Lemma 8 For all $0<r \in O$, the generalized tangent portfolio is given by the market portfolio:

$\theta_{m}(r)=\theta^{m}(r)$.

Proof. The market portfolio is

$\theta^{m}(r)=\frac{\operatorname{diag}[\Psi(r, \delta)] \phi^{m}}{\Psi\left(r, \delta^{m}\right)}$. Since both the generalized tangent portfolio and the market portfolio have unit length, and since diag $[\Psi(r, \delta)]$ is non-singular, it suffices to show that $\phi^{m}$ is proportional to

$\Sigma_{0}^{-1}\left(\mathbb{E}[\delta]-\frac{\Psi(r, \delta)}{\Psi(r, 1)}\right)$. In fact, with

$\mathbb{E}[\delta]-\frac{\Psi(r, \delta)}{\Psi(r, 1)}=\frac{r}{1-r} \mathbb{E}[\hat{c} \delta]=\frac{r}{1-r} \frac{1}{\sigma\left[\delta^{m}\right]} \Sigma_{0} \phi^{m}$ we ob-

tain $\phi^{m}=\frac{1-r}{r} \sigma\left[\delta^{m}\right] \Sigma_{0}^{-1}\left(\mathbb{E}[\delta]-\frac{\Psi(r, \delta)}{\Psi(r, 1)}\right)$ as desired.

In the light of this observation, by the two-fund separation theorem, all w-MPS risk averse investors will choose to hold a combination of the market portfolio and the risk free bond. By assumption A3, the market portfolio is finite, hence the efficient frontier exists. Let $\mathcal{I}(r)=\left\{a \theta^{m}(r): a \in \mathbb{R}\right\}$ be the efficient frontier that is composed of combinations of the risk free bond and the market portfolio. Therefore, for all $r$, the portfolio payoffs generated by efficient portfolios must coincide with the market subspace $\mathbb{D}_{0, m}$ that is spanned by the risk free bond and the market portfolio.

By assumption A5, there exists a utility representation on $\mathbb{D}_{0, m}$ that is given by $(x, y) \rightarrow U_{t}\left(x+y \delta^{m}\right)$. With initial wealth $W_{0}(r, t)=\Psi(r, e(t) \cdot \delta)$, the shares invested in the bond and the market portfolio can be expressed as $x=\frac{W_{0}(r, t)(1-a)}{\Psi(r, 1)}$ and $y=\frac{W_{0}(r, t) a}{\Psi\left(r, \delta^{m}\right)}$,

where $a$ is the proportion (of the wealth) invested in the market portfolio. Since, by assumption, $e(t) \cdot \delta>0$, the positivity of the pricing rule implies $W_{0}(r, t)>0$ for all $r \in O$. By the homotheticity of the utility function (Re: assumption A4), the optimal choice problem reduces to $\max _{a} V_{t}(a, r)$, where

$$
V_{t}(a, r) \stackrel{\Delta}{=} U_{t}\left(\frac{1-a}{\Psi(r, 1)}+\frac{a \delta^{m}}{\Psi\left(r, \delta^{m}\right)}\right) .
$$

For any $r$, assumptions A4 and A5 together imply that $(t, a) \rightarrow V_{t}(a, r)$ is $\Pi$-measurable in $t$ and continuous in $a \in \mathbb{R}$. By the strict-quasi-concavity of $a \rightarrow V_{t}(a, r)$, the optimal solution (if it exists) must be unique. Since no arbitrage condition is satisfied for $r \in O$ (Re: Lemma 6), by the Fundamental Theorem of Finance, the optimal solution exists (for $r \in O$ ). Let $\alpha(r, t)=\arg \max _{a \geq 0} V_{t}(a, r)$ be the optimal solution for $t$, where $r \in O$. Here, we restrict the number of shares invested in the market portfolio to be non-negative.

By the measurable maximum theorem ([29], Theorem 14.91), we have $t \rightarrow \alpha(r, t)$ to be $\Pi$-measurable. Lemma 9 below shows that $r \rightarrow \alpha(r, t)$ is continuous on $O$.

Lemma 9 For all $r \in O$ and $t \in T$, we have:

1. $\alpha(0, t)=0$.

2. If $\left\{r_{n}\right\}_{n=1}^{\infty} \subset O$ converges to $r^{*} \notin O$, then

$\lim _{n \rightarrow \infty} \alpha\left(r_{n}, t\right)=+\infty$.

3. $\alpha(\cdot, t): O \rightarrow \mathbb{R}_{+}$is continuous.

Proof. At $r=0$ the price of the market portfolio is given by $\mathbb{E}\left[\delta^{m}\right]$. The expected return of the market portfolio is thus given by the risk free interest rate $\left(\Psi^{-1}(0,1)=1\right)$. This implies that, for all $a$, the portfolio return $(1-a) \frac{1}{\Psi(0,1)}+a \frac{\delta^{m}}{\Psi\left(0, \delta^{m}\right)}$ is a w-MPS of the risk free return $\frac{1}{\Psi(0,1)}$. Therefore, all risk averse investors would optimally invest in the risk free bond with zero position in the market portfolio. That is, $\alpha(0, t)=0$ for all $t$.

To prove the second statement, let $\left\{r_{n}\right\}_{n=1}^{\infty} \subset O$ converge to $r^{*} \notin O$. Consider $\left\{\alpha\left(r_{n}, t\right)\right\}_{n=1}^{\infty}$. To show $\lim _{n \rightarrow \infty} \alpha\left(r_{n}, t\right)=+\infty$, suppose, to the contrary, that $\left\{\alpha\left(r_{n}, t\right)\right\}_{n=1}^{\infty}$ has a finite limit point given by $\alpha^{*} \geq 0$. Let $\left\{n_{k}\right\}_{k=1}^{\infty}$ be a convergent subsequence that con- 
verges to $\alpha^{*}$. We have: for all $a \in \mathbb{R}_{+}$, $V_{t}\left(a, r_{n}\right) \leq V_{t}\left(\alpha\left(r_{n}, t\right), r_{n}\right)$ for all $n$, particularly holds true for the subsequence $\left\{n_{k}\right\}_{k=1}^{\infty}$. Let $k \rightarrow \infty$, by continuity of $V_{t}$, we have: $V_{t}\left(a, r^{*}\right) \leq V_{t}\left(\alpha^{*}, r^{*}\right)$, which holds true for all $a$. Therefore, $\alpha^{*} \in \arg \max _{a \in \mathbb{R}_{+}} V_{t}\left(a, r^{*}\right)$. This, however, contradicts the emptiness of $G\left(r^{*}\right)$ at $r^{*}$. Therefore, we must have $\lim _{n \rightarrow \infty} \alpha\left(r_{n}, t\right)=+\infty$.

To show that $\alpha(\cdot, t): O \rightarrow \mathbb{R}_{+}$is continuous, it is sufficient to show that, for all $\left\{r_{n}\right\}_{n=1}^{\infty} \subset O$ converging to $r \in O$, the resulting sequence $\left\{\alpha\left(r_{n}, t\right)\right\}_{n=1}^{\infty}$ converges to $\alpha(r, t)$. Firstly, we show $\left\{\alpha\left(r_{n}, t\right)\right\}_{n=1}^{\infty}$ to constitute a bounded sequence. Suppose, without loss of generality, that $\lim _{n \rightarrow \infty} \alpha\left(r_{n}, t\right)=+\infty$. Let $\left\{a_{n}\right\}_{n=1}^{\infty} \subset \mathbb{R}_{+}$be an arbitrary sequence that converges to $\alpha(r, t)$. For any arbitrary $a>0$ let $x_{n}=\frac{a}{\alpha\left(r_{n}, t\right)}$ for all $n$. For $n$ sufficiently large, we have $x_{n} \in(0,1)$. The sequence $\left\{\left(1-x_{n}\right) a_{n}+x_{n} \alpha\left(r_{n}, t\right)\right\}_{n=1}^{\infty}$ converges to $\alpha(r, t)+a$. By the quasi-concavity of $V_{t}$, and by the optimality of $\alpha\left(r_{n}, t\right)$ we have

$V_{t}\left(\left(1-x_{n}\right) a_{n}+x_{n} \alpha\left(r_{n}, t\right), r_{n}\right) \geq V_{t}\left(a_{n}, r_{n}\right)$. Let $n \rightarrow \infty$, it yields $V_{t}(\alpha(r, t)+a, r) \geq V_{t}(\alpha(r, t), r)$. This contradicts the unique optimality of $\alpha(r, t)$ for the given $r \in O$.

Now, let $\alpha \geq 0$ be any finite limit point of $\left\{\alpha\left(r_{n}, t\right)\right\}_{n=1}^{\infty}$, and let $\left\{n_{k}\right\}_{k=1}^{\infty}$ be the convergent subsequence. We have, for all $a \in \mathbb{R}_{+}$and for all $k$, $V_{t}\left(a, r_{n_{k}}\right) \leq V_{t}\left(\alpha\left(r_{n_{k}}, t\right), r_{n_{k}}\right)$. Let $k \rightarrow \infty$, by continuity of $V_{t}$, we have $V_{t}(a, r) \leq V_{t}(\alpha, r)$, which holds for all $a$. Also, for $r \in O, \sup _{a \in \mathbb{R}_{+}} V_{t}(a, r)$ achieves its maximum uniquely at $\alpha(r, t)$. Therefore, we conclude that $\alpha=\alpha(r, t)$. This implies that $\left\{\alpha\left(r_{n}, t\right)\right\}_{n=1}^{\infty}$ has a unique limit point $\alpha(r, t)$; or, equivalently,

$\lim _{n \rightarrow \infty} \alpha\left(r_{n}, t\right)=\alpha(r, t)$. This ends the proof of the third statement.

As a necessary condition for the existence of a market equilibrium, the market clearing condition for the risky assets implies

$$
\int_{T} \alpha(r, t) \Psi(r, e(t) \cdot \delta) \Pi(\mathrm{d} t)=\Psi\left(r, \delta^{m}\right) ;
$$

that is, the aggregate investment in all risky assets equals to the value of the market portfolio. We have,

Lemma 10 There exists $0<r \in O$ that solves Equa- tion (13).

Proof. Let

$\alpha^{m}(r)=\frac{\int_{T} \alpha(r, t) \Psi(r, e(t) \cdot \delta) \Pi(\mathrm{d} t)}{\Psi\left(r, \delta^{m}\right)}, r \in O$. By

assumption A2 (the Feller's property) and by Lemma 9, we have $\alpha^{m}: O \rightarrow \mathbb{R}_{+}$to be continuous and to satisfy (i) $\alpha^{m}(0)=0$ and (ii) $\lim _{n \rightarrow \infty} \alpha^{m}\left(r_{n}\right)=\infty$ for all

$\left\{r_{n}\right\}_{n=1}^{\infty} \subset O$ converging to $r^{*}$. By the Intermediate Value Theorem, there exists an $r \in\left(0, r^{*}\right)$ such that $\alpha^{m}(r)=1$; or, equivalently,

$\int_{T} \alpha(r, t) \Psi(r, e(t) \cdot \delta) \Pi(\mathrm{d} t)=\Psi\left(r, \delta^{m}\right)$.

The validity of the main existence theorem, namely Theorem 2, can be concluded with the proof of Lemma 11:

Lemma 11 There exists an $r \in(0,1)$ such that $\pi(r)$ constitutes an equilibrium discount factor.

Proof. Let $0<r \in O$ be a solution to Equation (13). Lemma 8, together with Proposition 4, implies that $t$ 's optimal portfolio $\theta(t)$, for the given $r$, is proportional to the market portfolio. We write $\theta(t)=\alpha(r, t) \theta^{m}(r)$. With $W_{0}(r, t)=\Psi(r, e(t) \cdot \delta)$ we have

$$
\begin{aligned}
& \int_{T}(1-\alpha(r, t)) W_{0}(r, t) \Pi(\mathrm{d} t) \\
& =\Psi\left(r, \delta^{m}\right)-\int_{T} \alpha(r, t) \Psi(r, e(t) \cdot \delta) \Pi(\mathrm{d} t)=0 ;
\end{aligned}
$$

that is, the net borrowing in the risk free bond is zero. Moreover, for each risky asset $j$, we have

$$
\begin{aligned}
& \frac{\int_{T} \theta_{j}(t) W_{0}(r, t) \Pi(\mathrm{d} t)}{\Psi\left(r, \delta^{j}\right)} \\
& =\frac{\int_{T} \alpha(r, t) \Psi(r, e(t) \cdot \delta) \Pi(\mathrm{d} t)}{\Psi\left(r, \delta^{j}\right)} \theta_{j}^{m}(r)=\phi_{j}^{m} ;
\end{aligned}
$$

or, the total number of shares invested in $j$ equals to the number of shares outstanding for the security. Therefore, the pricing rule resulting from the discount factor $\pi(r)$ constitutes a market equilibrium. This concludes the proof.

\section{Concluding Remarks}

In this paper, we prove that the CAPM holds for economies with w-MPS risk averse investors. The CAPM model is shown to be valid without imposing any distributional restriction on asset returns and the number of tradable securities, and to be valid for economies with a continuum type of investors. This approach is compared to multi-factor models in the literature based on assumptions on the existence of some exogenous factors' structure in modeling asset returns. Our results suggest that, so long as investors exhibit w-MPS risk aversion, the relevance of all those factors that affect asset returns 
would all be summarized through the return of the market portfolio. This is true, at least, in equilibrium.

It is implicitly assumed in our analysis that investors endowments lie in the market span. For cases with nonspannable endowments as discussed in [17], the results on the validity of the CAPM and the existing proofs are still valid so long as the part of endowment, which is not market spannable is orthogonal to the market span.

\section{REFERENCES}

[1] M. Rothschild and J. Stiglitz, "Increasing Risk. I: A Definition," Journal of Economic Theory, Vol. 2, No. 3, 1970, pp. 225-243. http://dx.doi.org/10.1016/0022-0531(70)90038-4

[2] M. Rothschild and J. Stiglitz, "Increasing Risk. II: Its Economic Consequences," Journal of Economic Theory, Vol. 3, 1971, pp. 66-84. http://dx.doi.org/10.1016/0022-0531(71)90034-2

[3] D. Duffie, "Security Markets: Stochastic Models," Academic Press, 1988.

[4] C. Ma, "Advanced Asset Pricing Theory," Imperial College Press, 2011. http://dx.doi.org/10.1142/p745

[5] W. Sharpe, "Capital Asset Prices: A Theory of Capital Market Equilibrium under Conditions of Risk," Journal of Finance, Vol. 19, 1964, pp. 425-442.

[6] J. Lintner, "The Valuation of Risky Assets and the Selection of Risky Investment in Stock Portfolios and Capital Budgets," Review of Economics and Statistics, Vol. 47, No. 1, 1965, pp. 13-37. http://dx.doi.org/10.2307/1924119

[7] J. Mossin, "Equilibrium in a Capital Asset Market," Econometrica, Vol. 34, No. 4, 1966, pp. 768-783. http://dx.doi.org/10.2307/1910098

[8] G. Chamberlain, "A Characterization of the Distribution that Imply Mean-Variance Utility Functions," Journal of Economic Theory, Vol. 29, No. 1, 1983, pp. 185-201. http://dx.doi.org/10.1016/0022-0531(83)90129-1

[9] J. Owen and R. Rabinovitch, "On the Class of Elliptical Distributions and Their Applications to the Theory of Portfolio Choice," Journal of Finance, Vol. 38, No. 3, 1983, pp. 745-752. http://dx.doi.org/10.1111/j.1540-6261.1983.tb02499.x

[10] A. Löffler, "Variance Aversion Implies $\mu-\sigma^{2}$-Criterion," Journal of Economic Theory, Vol. 69, No. 2, 1996, pp. 532-539. http://dx.doi.org/10.1006/jeth.1996.0067

[11] R. J. Aumann, "Markets with a Continuum of Traders," Econometrica, Vol. 32, No. 1-2, 1964, pp. 39-50. http://dx.doi.org/10.2307/1913732

[12] R. J. Aumann, "Existence of Competitive Equilibria in Markets with a Continuum of Traders," Econometrica, Vol. 34, No. 1, 1966, pp. 1-17. http://dx.doi.org/10.2307/1909854

[13] D. Schmeidler, "Competitive Equilibria in Markets with a Continuum of Traders and Incomplete Preferences," Eco- nometrica, Vol. 37, No. 4, 1969, pp. 578-585. http://dx.doi.org/10.2307/1910435

[14] A. Mas-Colell, "An Equilibrium Existence Theorem without Complete or Transitive Preferences," Journal of Mathematical Economics, Vol. 1, No. 3, 1974, pp. 237-246. http://dx.doi.org/10.1016/0304-4068(74)90015-9

[15] D. Gale and A. Mas-Colell, "An Equilibrium Existence Theorem for a General Model without Ordered Preferences," Journal of Mathematical Economics, Vol. 2, No. 1, 1975, pp. 9-15. http://dx.doi.org/10.1016/0304-4068(75)90009-9

[16] R. A. Dana, "Existence, Uniqueness and Determinacy of Equilibrium in CAPM with a Riskless Asset," Journal of Mathematical Economics, Vol. 32, No. 2, 1999, pp. 167175. http://dx.doi.org/10.1016/S0304-4068(98)00050-0

[17] C. Hara, "Equilibrium Prices of the Market Portfolio in the CAPM with Incomplete Financial Markets," Working Paper, University of Cambridge, 2001.

[18] L. Nielsen, "Existence of Equilibrium in CAPM," Journal of Economic Theory, Vol. 52, No. 1, 1990, pp. 223231. http://dx.doi.org/10.1016/0022-0531(90)90076-V

[19] N. Sun and Z. Yang, "Existence of Equilibrium and Zero-Beta Pricing Formula in the Capital Asset Pricing Model with Heterogeneous Beliefs," Annals of Economics and Finance, Vol. 4, 2003, pp. 51-71.

[20] G. Chamberlain and M. Rothschild, "Arbitrage, Factor Structure, and Mean-Variance Analysis on Large Asset Markets," Econometrica, Vol. 50, 1983, pp. 1281-1304. http://dx.doi.org/10.2307/1912275

[21] F. Black, "Capital Market Equilibrium with Restricted Borrowing," Journal of Business, Vol. 45, No. 3, 1972, pp. 444-455. http://dx.doi.org/10.1086/295472

[22] H. Markowitz, "Portfolio Selection," Journal of Finance, Vol. 7, 1952, pp. 77-99.

[23] H. Markowitz, "Portfolio Selection," John Wiley and Sons, Inc., New York, 1959.

[24] J. Tobin, "Liquidity Preference and Behavior towards Risk," Review of Economic Studies, Vol. 25, 1958, pp. 65-86. http://dx.doi.org/10.2307/2296205

[25] S. Ross, "Mutual Fund Separation in Financial TheoryThe Separating Distributions," Journal of Economic Theory, Vol. 17, 1978, pp. 254-286. http://dx.doi.org/10.1016/0022-0531(78)90073-X

[26] C. F. Huang and R. Litzenberger, "Foundations for Financial Economics," Prentice Hall, Inc., 1988.

[27] J. Berk, "Necessary and Sufficient Conditions for the CAPM," Journal of Economic Theory, Vol. 73, 1997, pp. 245-257. http://dx.doi.org/10.1006/jeth.1996.2218

[28] P. Dybvig and S. Ross, "Arbitrage," In: The New Palgrave: A Dictionary of Economics, The MacMillan Press Limited, 1987.

[29] C. Aliprantis and K. Border, "Infinite Dimensional Analysis," Springer-Verlag, 1994. http://dx.doi.org/10.1007/978-3-662-03004-2 